\title{
A Category of Higher-Dimensional Automata
}

\author{
Ulrich Fahrenberg \\ Dept. of Mathematical Sciences, Aalborg University, Denmark \\ uli@math.aau.dk
}

\begin{abstract}
We show how parallel composition of higher-dimensional automata (HDA) can be expressed categorically in the spirit of Winskel \& Nielsen. Employing the notion of computation path introduced by van Glabbeek, we define a new notion of bisimulation of HDA using open maps. We derive a connection between computation paths and carrier sequences of dipaths and show that bisimilarity of HDA can be decided by the use of geometric techniques.
\end{abstract}

Keywords: Higher-dimensional automata, bisimulation, open maps, directed topology, fibrations.

\section{Introduction}

In his invited talk at the 2004 ExPRESS workshop, van Glabbeek 11] put higherdimensional automata (HDA) on top of a hierarchy of models for concurrency. In this article we develop a categorical framework for expressing constructions on HDA, building on work by Goubault in 12,13.

Following up on a concluding remark in 13, we introduce a notion of bisimulation of HDA, both as a relation and using open maps [19. Our notion differs from the ones introduced by van Glabbeek [10] and Cattani-Sassone 4 .

Employing recent developments by Fajstrup [8, we show that bisimilarity of HDA is equivalent to a certain dipath-lifting property, which can be attacked using (directed) homotopy techniques. This confirms a prediction from [13.

Due to space limitations, we had to omit some of the more technical points in this paper. An extended version is published in [6].

The author is indebted to Eric Goubault and Emmanuel Haucourt for many valuable discussions during his visit at CEA in Paris, and to Lisbeth Fajstrup and Martin Raussen at the Department of Mathematical Sciences in Aalborg.

\section{Cubical Sets}

Cubical sets were introduced by Serre in 22 and have a variety of applications in algebraic topology, both in homology, cf. 20, and in homotopy theory, cf. 2. 5, 18. Compared to the more well-known simplicial sets, they have the distinct advantage that they have a natural sense of (local) direction induced by the order 
on the unit interval. This makes them well-suited for applications in concurrency theory, cf. 9.

A precubical set is a graded set $X=\left\{X_{n}\right\}_{n \in \mathbb{N}}$ together with mappings $\delta_{i(n)}^{\nu}$ : $X_{n} \rightarrow X_{n-1}, i=1, \ldots, n, \nu=0,1$, satisfying the precubical identity

$$
\delta_{i}^{\nu} \delta_{j}^{\mu}=\delta_{j-1}^{\mu} \delta_{i}^{\nu} \quad(i<j)
$$

These are called face maps, and if $x=\delta_{i_{1}}^{\nu_{1}} \cdots \delta_{i_{n}}^{\nu_{n}} y$ for some cubes $x, y$ and some (possibly empty) sequences of indices, then $x$ is called a face of $y$. If all $\nu_{i}=0$, $x$ is said to be a lower face of $y$; if all $\nu_{i}=1, x$ is an upper face of $y$.

As above, we shall omit the subscript $(n)$ in $\delta_{i(n)}^{\nu}$ whenever possible. Elements of $X_{n}$ are called $n$-cubes.

A cubical set is a precubical set $X$ together with mappings $\epsilon_{i(n)}: X_{n} \rightarrow X_{n+1}$, $i=1, \ldots, n+1$, such that

$$
\epsilon_{i} \epsilon_{j}=\epsilon_{j+1} \epsilon_{i} \quad(i \leq j) \quad \delta_{i}^{\nu} \epsilon_{j}= \begin{cases}\epsilon_{j-1} \delta_{i}^{\nu} & (i<j) \\ \epsilon_{j} \delta_{i-1}^{\nu} & (i>j) \\ \mathrm{id} & (i=j)\end{cases}
$$

These are called degeneracies, and equations (1) and (2) together form the cubical identities.

The standard example of a cubical set is the singular cubical complex of a topological space, cf. 20]: If $X$ is a topological space, let $S_{n} X=\operatorname{Top}\left(I^{n}, X\right)$, the set of all continuous maps $I^{n} \rightarrow X$, where $I$ is the unit interval. If the maps $\delta_{i}^{\nu}$ and $\epsilon_{i}$ are given by

$$
\begin{aligned}
\delta_{i}^{\nu} f\left(t_{1}, \ldots, t_{n-1}\right) & =f\left(t_{1}, \ldots, t_{i-1}, \nu, t_{i}, \ldots, t_{n-1}\right) \\
\epsilon_{i} f\left(t_{1}, \ldots, t_{n}\right) & =f\left(t_{1}, \ldots, \hat{t}_{i}, \ldots, t_{n}\right)
\end{aligned}
$$

(the notation $\hat{t}_{i}$ means that $t_{i}$ is omitted) then $S X=\left\{S_{n} X\right\}$ is a cubical set.

Morphisms of (pre)cubical sets are required to commute with the structure maps, i.e. if $X, Y$ are two (pre)cubical sets, then a morphism $f: X \rightarrow Y$ is a sequence of mappings $f=\left\{f_{n}: X_{n} \rightarrow Y_{n}\right\}$ that fulfill the first, respectively both, of the equations

$$
\delta_{i}^{\nu} f_{n}=f_{n-1} \delta_{i}^{\nu} \quad \epsilon_{i} f_{n}=f_{n+1} \epsilon_{i}
$$

This defines two categories, pCub and Cub, both of which are presheaf categories over certain small categories of elementary cubes, cf. [17, hence they are Cartesian closed, complete, and cocomplete. The forgetful functor

$$
\mathrm{Cub} \longrightarrow \mathrm{pCub}
$$

has a left adjoint, providing us with a "free" functor in the opposite direction which we shall denote $F$.

A (pre)cubical set $X=\left\{X_{n}\right\}$ is said to be $k$-dimensional if $X_{n}=\emptyset$ for $n>k$. The full subcategories of $k$-dimensional objects in our cubical categories are denoted $\mathrm{pCub}^{k}$ respectively $\mathrm{Cub}^{k}$. The free-forgetful adjunction above passes to the $k$-dimensional categories. 


\section{Product and Tensor Product}

The product of two (pre)cubical sets is given by

$$
(X \times Y)_{n}=X_{n} \times Y_{n}
$$

with face maps and degeneracies defined component-wise. This is a product in the categorical sense. A (pre)cubical relation between (pre)cubical sets $X, Y$ is a (pre)cubical subset of the product $X \times Y$.

The tensor product of two precubical sets $Z=X \otimes Y$ is given by

$$
Z_{n}=\bigsqcup_{p+q=n} X_{p} \times Y_{q}
$$

with face maps

$$
\delta_{i}^{\alpha}(x, y)=\left\{\begin{array}{ll}
\left(\delta_{i}^{\alpha} x, y\right) & (i \leq p) \\
\left(x, \delta_{i-p}^{\alpha} y\right) & (i \geq p+1)
\end{array} \quad(x, y) \in X_{p} \times Y_{q}\right.
$$

The category Cub inherits this tensor product, however some identifications have to be made to get well-defined degeneracy maps, cf. 3]. The tensor product of two cubical sets $Z=X \otimes Y$ is then given by

$$
Z_{n}=\left(\bigsqcup_{p+q=n} X_{p} \times Y_{q}\right) / \sim_{n}
$$

where $\sim_{n}$ is the equivalence relation generated by, for all $(x, y) \in X_{r} \times Y_{s}$, $r+s=n-1$, letting $\left(\epsilon_{r+1} x, y\right) \sim_{n}\left(x, \epsilon_{1} y\right)$. If $x \otimes y$ denotes the equivalence class of $(x, y) \in X_{p} \times Y_{q}$ under $\sim_{n}$, the face maps and degeneracies of $Z$ are given by

$$
\delta_{i}^{\alpha}(x \otimes y)=\left\{\begin{array}{ll}
\delta_{i}^{\alpha} x \otimes y & (i \leq p) \\
x \otimes \delta_{i-p}^{\alpha} y & (i \geq p+1)
\end{array} \quad \epsilon_{i}(x \otimes y)= \begin{cases}\epsilon_{i} x \otimes y & (i \leq p+1) \\
x \otimes \epsilon_{i-p} y & (i \geq p+1)\end{cases}\right.
$$

\section{Transition Systems}

We shall construct our category of higher-dimensional automata as a special arrow category in Cub. To warm up, we include a section on how transition systems can be understood as an arrow category in $\mathrm{Cub}^{1}$, the category of digraphs. Though our exposition differs considerably from the standard one, see e.g. [23, the end result is basically the same.

A digraph is a 1-dimensional cubical set, i.e. a pair of sets $\left(X_{1}, X_{0}\right)$ together with face maps $\delta^{0}, \delta^{1}: X_{1} \rightarrow X_{0}$ and a degeneracy mapping $\epsilon=\epsilon_{1}: X_{0} \rightarrow X_{1}$ such that $\delta^{0} \epsilon=\delta^{1} \epsilon=$ id. Morphisms of digraphs $\left(X_{1}, X_{0}\right),\left(Y_{1}, Y_{0}\right)$ are thus mappings $f=\left(f_{1}, f_{0}\right)$ commuting with the face and degeneracy mappings. A predigraph is a 1-dimensional precubical set. Note that we allow both loops and multiple edges in our digraphs. 
The category of digraphs has a terminal object $*$ consisting of a single vertex and the degeneracy edge on that vertex. A transition system is a digraph which is freely generated by a predigraph together with a specified initial point, hence the category of transition systems is $\left\langle * \downarrow F \mathrm{pCub}^{1}\right\rangle$, the comma category of digraphs freely generated by predigraphs under $*$. In the spirit of 23 , passing from a predigraph to the digraph freely generated by it means that we add idle loops to each vertex, hence allowing for transition system morphisms which collapse transitions.

As for labeling transition systems, we note that there is an isomorphism between the category of finite sets and the full subcategory of $\mathrm{pCub}^{1}$ induced by finite one-point predigraphs, given by mapping a finite set $\Sigma$ to the onepoint predigraph with edge set $\Sigma$. Identifying finite sets with the digraphs freely generated by their associated predigraphs, we define a labeled transition system over $\Sigma$ to be a digraph morphism $\lambda:\left\langle * \downarrow F \mathrm{pCub}^{1}\right\rangle \rightarrow \Sigma$ which is induced by a predigraph morphism. This last convention is to ensure that idle loops are labeled with the idle label $\epsilon *$.

Say that a morphism $\lambda \in \mathrm{Cub}^{1}$ is non-contracting if $\lambda a=\epsilon *$ implies $a=\epsilon \delta^{0} a$ for all edges $a$, and note that if the source and target of $\lambda$ are freely generated by precubical sets, then $\lambda$ is non-contracting if and only if it is in the image of the free functor $\mathrm{pCub}^{1} \rightarrow \mathrm{Cub}^{1}$.

For morphisms between labeled transition systems we need to allow functions that map labels to "nothing," i.e. partial alphabet functions. The category of finite sets with partial mappings is isomorphic to the full subcategory $\Sigma$ of Cub ${ }^{1}$ induced by digraphs freely generated by finite one-point predigraphs. Hence we can define the category of labeled transition systems to be the non-contracting comma-arrow category $\left\langle * \downarrow F \mathrm{pCub}^{1} \rightrightarrows \Sigma\right\rangle$, with objects pairs of morphisms - the second one non-contracting

$$
* \longrightarrow X \Longrightarrow \Sigma
$$

and morphisms pairs of arrows making the following square commute:

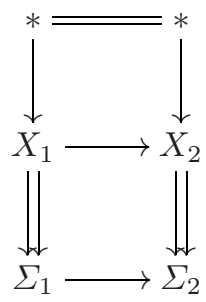

We shall always visualise non-contracting morphisms by double arrows.

Note that our transition systems have the special feature that there can be more than one transition with a given label between a pair of edges; in the terminology of 23] they are not extensional. Except for that, our definition is in accordance with the standards.

To express parallel composition of transition systems, we follow the approach of [23] and use a combination of product, relabeling and restriction. In our context, the product of two transition systems $* \rightarrow X_{1} \rightarrow \Sigma_{1}$, $* \rightarrow X_{2} \rightarrow \Sigma_{2}$ is the 
transition system $* \longrightarrow X_{1} \times X_{2} \stackrel{\lambda}{\longrightarrow} \Sigma_{1} \times \Sigma_{2}$, where the arrow $\lambda$ is given by the universal property of the product $\Sigma_{1} \times \Sigma_{2}$. We note that, indeed, the product of two one-point digraphs with edge sets $\Sigma_{1}$ respectively $\Sigma_{2}$ is again a one-point digraph, with edge set

$$
\left\{(a, b),(a, \epsilon *),(\epsilon *, b) \mid a \in \Sigma_{1}, b \in \Sigma_{2}\right\}
$$

One easily shows $\lambda$ to be non-contracting, and the so-defined product is in fact the categorical product in the category $\left\langle * \downarrow F \mathrm{pCub}^{1} \rightrightarrows \Sigma\right\rangle$.

A relabeling of a transition system is a non-contracting alphabet morphism under the identity, i.e. an arrow in $\left\langle * \downarrow F \mathrm{pCub}^{1} \rightrightarrows \Sigma\right\rangle$ of the form

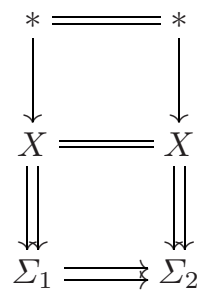

Restriction of transition systems is defined using pullbacks; given a transition system $* \rightarrow X_{2} \rightarrow \Sigma_{2}$ and a mapping $\sigma: \Sigma_{1} \rightarrow \Sigma_{2}$, we define the restriction of $X_{2}$ to $\Sigma_{1}$ by the pullback

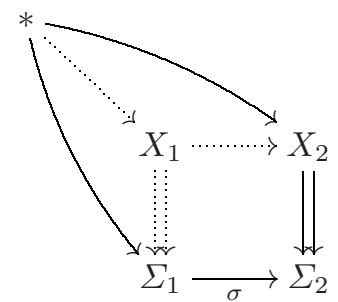

where the mapping $* \rightarrow \Sigma_{1}$ is uniquely determined as $\Sigma_{1}$ is a one-point digraph. It is not difficult to show that the so-defined morphism $X_{1} \rightarrow \Sigma_{1}$ is in fact non-contracting.

\section{Higher-Dimensional Automata}

The category Cub has a terminal object $*$ consisting of a single point and all its higher-dimensional degeneracies. The category of higher-dimensional automata is the comma category $\langle * \downarrow F \mathrm{pCub}\rangle$, with objects cubical sets freely generated by precubical sets with a specified initial 0-cube.

For labeling HDA, we follow the approach laid out in 12, 13. We assume the finite set $\Sigma$ of labels to be totally ordered and define a precubical set $! \Sigma^{\prime}$ as follows: $! \Sigma_{0}^{\prime}=\{*\}, ! \Sigma_{n}^{\prime}$ is the set of (not necessarily strictly) increasing sequences of length $n$ of elements of $\Sigma$, and

$$
\delta_{i(n)}^{\alpha}\left(x_{1}, \ldots, x_{n}\right)=\left(x_{1}, \ldots, \hat{x}_{i}, \ldots, x_{n}\right)
$$


Then we let $! \Sigma$ be the free cubical set on $! \Sigma^{\prime}$.

Let $! \Sigma$ be the full subcategory of Cub induced by the cubical sets $! \Sigma$ as above. We show in [6] that ! $\Sigma$, like the category $\Sigma$ in the preceding section, is isomorphic to the category of finite sets and partial (and not necessarily order-preserving) mappings.

Define a morphism $f: X \rightarrow Y$ of cubical sets to be non-contracting if $f(x)=\epsilon_{i} \delta_{i}^{0} f(x)$ implies $x=\epsilon_{i} \delta_{i}^{0} x$ for all $x \in X_{n}, n \in \mathbb{N}, i=1, \ldots, n$. Note again that if the cubical sets $X, Y$ are freely generated by precubical sets, then a morphism $f: X \rightarrow Y$ is non-contracting if and only if it is the image of a precubical morphism under the free functor.

The category of labeled higher-dimensional automata is then defined to be $\langle * \downarrow F \mathrm{pCub} \rightrightarrows ! \Sigma\rangle$, with objects $* \longrightarrow X \Longrightarrow ! \Sigma$ and morphisms commutative diagrams

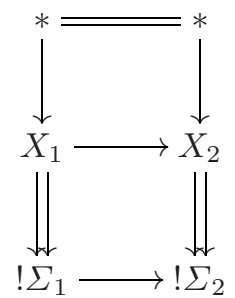

Note that by this construction, the label of an $n$-cube is the ordered $n$-tuple of the labels of all its 1-faces.

\section{Constructions on HDA}

As in [12, we replace the product of transition systems by the tensor product of higher-dimensional automata. The tensor product of two HDA $* \rightarrow X_{1} \stackrel{\lambda}{\longrightarrow} ! \Sigma_{1}$, $* \rightarrow X_{2} \stackrel{\mu}{\longrightarrow} ! \Sigma_{2}$ is defined to be

$$
* \longrightarrow X_{1} \otimes X_{2} \stackrel{\lambda \otimes \mu}{\longrightarrow} ! \Sigma_{1} \otimes ! \Sigma_{2}
$$

The following lemma, where $\Sigma_{1} \uplus \Sigma_{2}$ denotes the disjoint union of $\Sigma_{1}$ and $\Sigma_{2}$ with the order induced by declaring $\Sigma_{1}<\Sigma_{2}$, ensures that this in in fact a HDA:

Lemma 1. Given alphabets $\Sigma_{1}, \Sigma_{2}$, then $! \Sigma_{1} \otimes ! \Sigma_{2}=!\left(\Sigma_{1} \uplus \Sigma_{2}\right)$.

For relabeling HDA we use non-contracting morphisms under the identity, and we note that if $g$ is defined by the diagram

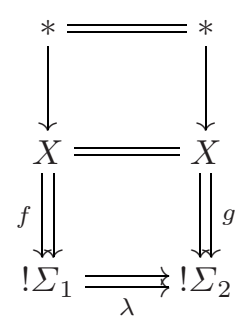

then non-contract ability of $g$ follows from $f$ and $\lambda$ being non-contracting. 
If we want to express the tensor product of two HDA $* \rightarrow X \rightarrow ! \Sigma_{1}, * \rightarrow$ $Y \rightarrow ! \Sigma_{2}$ with non-disjoint alphabets $\Sigma_{1}, \Sigma_{2}$, we can do so by following the tensor product above with a relabeling $! \Sigma_{1} \otimes ! \Sigma_{2} \rightarrow !\left(\Sigma_{1} \cup \Sigma_{2}\right)$ induced by the natural projection $\Sigma_{1} \uplus \Sigma_{2} \rightarrow \Sigma_{1} \cup \Sigma_{2}$ (which is not necessarily order-preserving). This projection is a total alphabet morphism, hence the relabeling map is indeed non-contracting.

For restrictions we again use pullbacks:

Proposition 1. Given a higher-dimensional automaton $* \rightarrow X_{2} \rightarrow ! \Sigma_{2}$ and an injective mapping $! \Sigma_{1} \rightarrow ! \Sigma_{2}$, then $* \rightarrow X_{1} \rightarrow ! \Sigma_{1}$ as defined by the pullback diagram

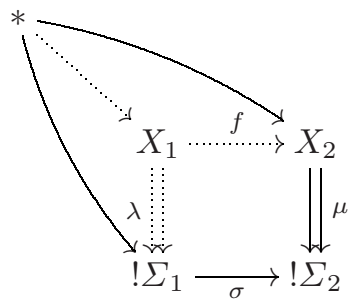

is again a higher-dimensional automaton.

The arrow $* \rightarrow ! \Sigma_{1}$ is uniquely determined as $! \Sigma_{1}$ has only one cube in dimension zero. We will need the injectivity of $\sigma$ later, to show that our to-bedefined notion of bisimilarity is respected by restrictions.

\section{Bisimulation}

In this section we fix a labeling cubical set $L$ and work in the non-contracting double comma category $\langle * \downarrow F$ pCub $\downarrow L\rangle$ of HDA over $L$. The morphisms

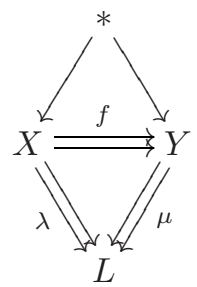

in this category respect labelings, hence they are non-contracting themselves: If $f(x)=\epsilon_{i} \delta_{i}^{0} f(x)$ for some $x \in X$ and some $i$, then $\lambda(x)=\mu(f(x))=\epsilon_{i} \delta_{i}^{0} \lambda(x)$ and thus $x=\epsilon_{i} \delta_{i}^{0} x$.

A computation path, cf. [10, in a precubical set $X$ is a finite sequence $\left(x_{1}, \ldots\right.$, $x_{n}$ ) of cubes of $X$ such that for each $k=1, \ldots, n-1$, either $x_{k}=\delta_{i}^{0} x_{k+1}$ or $x_{k+1}=\delta_{i}^{1} x_{k}$ for some $i$. A computation path $\left(x_{1}, \ldots, x_{n}\right)$ is said to be acyclic if there are no other relations between the $x_{i}$ than the ones above. A rooted computation path in a $\mathrm{HDA} * \stackrel{i}{\longrightarrow} X$ is a computation path $\left(i *, \ldots, x_{n}\right)$, and a 


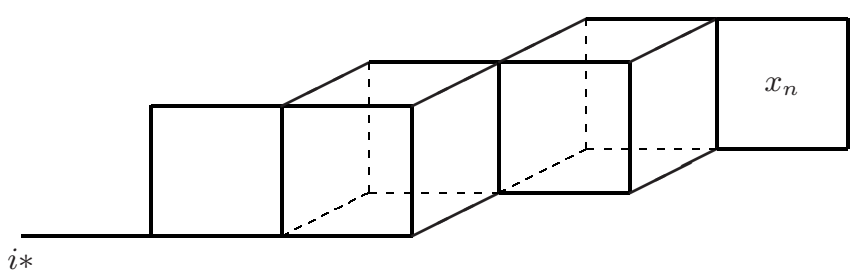

Fig. 1. An acyclic rooted computation path which ends in a 2-cube $x_{n}$

cube $x$ of the HDA is said to be reachable if there is a rooted computation path $(i *, \ldots, x)$. Figure 1 shows an example of an acyclic rooted computation path.

We say that a precubical set $X$ is a computation path if there is a computation path $\left(x_{1}, \ldots, x_{n}\right)$ of cubes in $X$ such that all other cubes in $X$ are faces of one of the $x_{i}$, and similarly for acyclic computation paths. An elementary computation step is an inclusion $\left(x_{1}, \ldots, x_{n}\right) \hookrightarrow\left(x_{1}, \ldots, x_{n}, x_{n+1}\right)$ of computation paths.

Let CPath be the full subcategory of the category of HDA induced by the acyclic rooted computation paths, then it is not difficult to see that any morphism in CPath is a finite composite of elementary computation steps and isomorphisms.

Following the terminology of [19], we say that a morphism $f: X \rightarrow Y$ is CPath-open if it has the right-lifting property with respect to morphisms in CPath. That is, we require that for any morphism $m: P \rightarrow Q \in$ CPath and any commutative diagram as below, there exists a morphism $r$ filling in the diagram

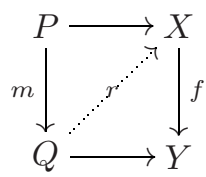

Lemma 2. A morphism $f: X \rightarrow Y$ is CPath-open if and only if it satisfies the property that for any reachable $x \in X$ and for any $z^{\prime} \in Y$ such that $f(x)=\delta_{i}^{0} z^{\prime}$ for some $i$, there is a $z \in X$ such that $x=\delta_{i}^{0} z$ and $z^{\prime}=f(z)$.

Following established terminology, this could be called a "higher-dimensional zig-zag property."

This suggests the following definition of bisimulation of HDA: Given two HDA $* \stackrel{i}{\longrightarrow} X \stackrel{\lambda}{\longrightarrow} L, * \stackrel{j}{\longrightarrow} Y \stackrel{\mu}{\longrightarrow} L$ over the same alphabet, then a bisimulation of $X$ and $Y$ is a cubical relation $R \subseteq X \times Y$ which respects initial states and labelings, i.e. $(i *, j *) \in R_{0}$, and if $(x, y) \in R$ then $\lambda x=\mu y$; and for all reachable $x \in X, y \in Y$ such that $(x, y) \in R$,

- if $x=\delta_{i}^{0} z$ for some $z$, then $y=\delta_{i}^{0} z^{\prime}$ for some $z^{\prime}$ so that $\left(z, z^{\prime}\right) \in R$,

- if $y=\delta_{i}^{0} z^{\prime}$ for some $z^{\prime}$, then $x=\delta_{i}^{0} z$ for some $z$ so that $\left(z, z^{\prime}\right) \in R$.

Note that bisimilarity is indeed an equivalence relation. 
Proposition 2. Two HDA Y, $Z$ are bisimilar if and only if there is a span of CPath-open maps $Y \leftarrow X \rightarrow Z$.

Note that when restricted to labeled transition systems, bisimulation of HDA is equivalent to strong bisimulation 21, the only difference being that strong bisimulation requires the existence of corresponding transitions, whereas HDAbisimulation actually specifies a correspondence.

\section{Bisimulation Is a Congruence}

We show that bisimulation is a congruence with respect to the constructions on HDA introduced in Section 6. For relabelings this is clear, and for tensor product we have the following lemma.

Lemma 3. Given CPath-open morphisms $f \in\langle * \downarrow F \mathrm{pCub} \downarrow L\rangle, g \in\langle * \downarrow F \mathrm{pCub} \downarrow$ $\downarrow M\rangle$, then $f \otimes g \in\langle * \downarrow F \mathrm{pCub} \downarrow L \otimes M\rangle$ is again CPath-open.

Hence if we have spans of CPath-open morphisms $Y_{1} \stackrel{f_{1}}{\longleftarrow} X_{1} \stackrel{g_{1}}{\longrightarrow} Z_{1}, Y_{2} \stackrel{f_{2}}{\longleftarrow}$ $X_{2} \stackrel{g_{2}}{\longrightarrow} Z_{2}$, then $Y_{1} \otimes Y_{2}$ and $Z_{1} \otimes Z_{2}$ are bisimilar via the span of CPath-open morphisms $Y_{1} \otimes Y_{2} \stackrel{f_{1} \otimes f_{2}}{\longleftarrow} X_{1} \otimes X_{2} \stackrel{g_{1} \otimes g_{2}}{\longrightarrow} Z_{1} \otimes Z_{2}$.

Congruency of bisimilarity with respect to restriction is implied by the next lemma.

Lemma 4. Given a CPath-open morphism $f: X \rightarrow Y \in\langle * \downarrow F \mathrm{pCub} \downarrow L\rangle$ and a non-contracting injective morphism $\sigma: L^{\prime} \rightarrow L$, then the unique morphism $f^{\prime}: X^{\prime} \rightarrow Y^{\prime}$ defined by the double pullback diagram

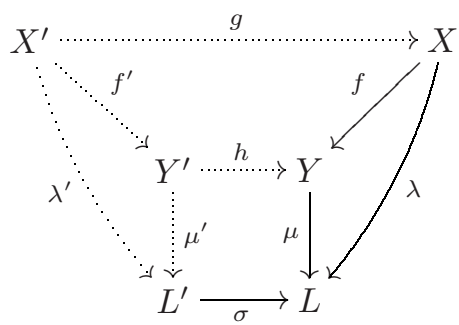

is again CPath-open.

Hence if $Y, Z \in\langle * \downarrow F \mathrm{pCub} \Downarrow L\rangle$ are bisimilar via a span of CPath-open maps $Y \leftarrow X \rightarrow Z$, the above lemma yields a span of CPath-open maps $Y^{\prime} \leftarrow X^{\prime} \rightarrow Z^{\prime}$ of their restrictions to $L^{\prime}$.

\section{Geometric Realisation of Precubical Sets}

We want to relate CPath-openness of a morphism of higher-dimensional automata to a geometric property of the underlying precubical sets. In order to do that, we need to recall some of the technical apparatus developed in 9,8 . 
The geometric realisation of a precubical set $X$ is the topological space

$$
|X|=\bigsqcup_{n \in \mathbb{N}} X_{n} \times[0,1]^{n} / \equiv
$$

where the equivalence relation $\equiv$ is induced by identifying

$$
\left(\delta_{i}^{\nu} x ; t_{1}, \ldots, t_{n-1}\right) \equiv\left(x ; t_{1}, \ldots, t_{i-1}, \nu, t_{i}, \ldots, t_{n-1}\right)
$$

for all $x \in X_{n}, n \in \mathbb{N}, i=1, \ldots, n, \nu=0,1, t_{i} \in[0,1]$. Geometric realisation is turned into a functor from $\mathrm{pCub}$ to Top by mapping $f: X \rightarrow Y \in \mathrm{pCub}$ to the function $|f|:|X| \rightarrow|Y|$ defined by

$$
|f|\left(x ; t_{1}, \ldots, t_{n}\right)=\left(f(x) ; t_{1}, \ldots, t_{n}\right)
$$

This is similar to the well-known geometric realisation functor from simplicial sets to topological spaces, cf. [1].

Given $x \in X_{n} \in X$, we denote its image in the geometric realisation by $|x|=\left\{\left(x ; t_{1}, \ldots, t_{n}\right) \mid t_{i} \in[0,1]\right\} \subseteq|X|$. The carrier, carr $z$, of a point $z \in|X|$ is $z$ itself if $z \in X_{0}$, or else the unique cube $x \in X$ such that $z \in$ int $|x|$, the interior of $|x|$. The star of $z$ is the open set

$$
\text { St } z=\left\{z^{\prime} \in|X| \mid \operatorname{carr} z \triangleleft \operatorname{carr} z^{\prime}\right\}
$$

There is a natural order on the cubes $[0,1]^{n}$ which is "forgotten" in the transition $\mathrm{pCub} \longrightarrow$ Top. One can recover some of this structure by instead defining functors from pCub to the d-spaces or the spaces with distinguished cubes of M. Grandis [14, 15, 16, however here we take a different approach as laid out in 9 .

Given a precubical set $X$ and $x, y \in X$, we write $x \triangleleft y$ if $x$ is a face of $y$. This defines a preorder $\triangleleft$ on $X$. If $x$ is a lower face of $y$ we write $x \triangleleft^{-} y$, if it is an upper face we write $x \triangleleft^{+} y$. The precubical set $X$ is said to be locally finite if the set $\{y \in X \mid x \triangleleft y\}$ is finite for all $x \in X_{0}$.

Define a precubical set $X$ to be non-selflinked if $\delta_{i}^{\nu} x=\delta_{j}^{\mu} x$ implies $i=j$, $\nu=\mu$ for all $x \in X, i, j \in \mathbb{N}_{+}, \nu, \mu \in\{0,1\}$. Note [9-Lemma 6.16]: If $x \triangleleft y$ in a non-selflinked precubical set, then there are unique sequences $\nu_{1}, \ldots, \nu_{\ell}$, $i_{1}<\cdots<i_{\ell}$ such that $x=\delta_{i_{1}}^{\nu_{1}} \cdots \delta_{i_{\ell}}^{\nu \ell} y$.

The geometric realisation of a non-selflinked precubical set contains no selfintersections; if $\left(x, s_{1}, \ldots, s_{n}\right) \equiv\left(x, t_{1}, \ldots, t_{n}\right)$, then $s_{i}=t_{i}$ for all $i=1, \ldots, n$. By [9-Thm. 6.27], the geometric realisation of a non-selflinked precubical set is a local po-space; a Hausdorff topological space with a relation $\leq$ which is reflexive, antisymmetric, and locally transitive, i.e. transitive in each $U_{\alpha}$ for some collection $\mathcal{U}=\left\{U_{\alpha}\right\}$ of open sets covering $X$. In our case, the relation $\leq$ is induced by the natural partial orders on the unit cubes $[0,1]^{n}$, and a covering $\mathcal{U}$ is given by the stars St $|x|$ of all vertices $x \in X_{0}$.

A dimap between local po-spaces $\left(X, \leq_{X}\right),\left(Y, \leq_{Y}\right)$ is a continuous mapping $f: X \rightarrow Y$ which is locally increasing: for any $x \in X$ there is an open neighbourhood $U \ni x$ such that for all $x_{1} \leq_{X} x_{2} \in U, f\left(x_{1}\right) \leq_{Y} f\left(x_{2}\right)$. Local po-spaces 
and dimaps form a category IpoTop, and by [9-Prop. 6.38], geometric realisation is a functor from non-selflinked precubical sets to local po-spaces.

Let $\vec{I}$ denote the unit interval $[0,1]$ with the natural (total) order, and define a dipath in a local po-space $(S, \leq)$ to be a dimap $p: \vec{I} \rightarrow S$. We recall 8 Def. 2.17]: Given a locally finite precubical set $X$ and a dipath $p: \vec{I} \rightarrow|X|$, then there exists a partition of the unit interval $0=t_{1} \leq \cdots \leq t_{k+1}=1$ and a unique sequence $x_{1}, \ldots, x_{k} \in X$ such that

$-x_{i} \neq x_{i+1}$

$-t \in\left[t_{i}, t_{i+1}\right]$ implies $p(t) \in\left|x_{i}\right|$

$-t \in] t_{i}, t_{i+1}\left[\right.$ implies carr $p(t)=x_{i}$

$-\operatorname{carr} p\left(t_{i}\right) \in\left\{x_{i-1}, x_{i}\right\}$

The sequence $\left(x_{1}, \ldots, x_{k}\right)$ is called the carrier sequence of the dipath $p$, and we shall denote it by carrs $p$. It can be shown, cf. [8-Lemma 3.2], that for all $i=2, \ldots, n$, either $x_{i-1} \triangleleft^{-} x_{i}$ or $x_{i} \triangleleft^{+} x_{i-1}$. Note that the definition in 8 makes an extra assumption on $X$ which, in fact, is not necessary. Figure 2 shows an example of a carrier sequence.

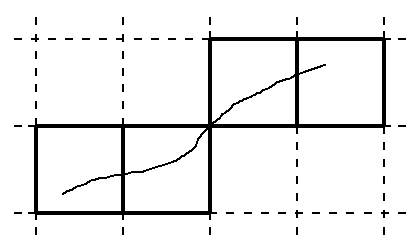

Fig. 2. A dipath and its carrier sequence

In general we call a sequence of cubes $\left(x_{1}, \ldots, x_{n}\right)$ a carrier sequence if $x_{i-1} \triangleleft^{-}$ $x_{i}$ or $x_{i} \triangleleft^{+} x_{i-1}$ for all $i=2, \ldots, n$. Note that computation paths are carrier sequences, and conversely, that carrier sequences can be turned into computation paths by adding in some intermediate cubes. The next lemma shows that any carrier sequence actually is the carrier sequence of a dipath.

Lemma 5. Given a carrier sequence $\left(x_{1}, \ldots, x_{n}\right)$ in a locally finite non-selflinked precubical set $X$ and $z \in$ int $\left|x_{n}\right|$, there exists a dipath $p: \vec{I} \rightarrow|X|$ such that $\operatorname{carrs} p=\left(x_{1}, \ldots, x_{n}\right)$ and $p(1)=z$.

We can similarly fix $z \in$ int $\left|x_{1}\right|$ and get a dipath $p$ with $p(0)=z$, but we will only need the former case. We shall also need the following two technical lemmas.

Lemma 6. Given locally finite non-selflinked precubical sets $X, Y$, a morphism $f: X \rightarrow Y$, and a dipath $p: \vec{I} \rightarrow|X|$, then $\operatorname{carrs}(|f| \circ p)=f(\operatorname{carrs} p)$.

Note that, taking $p$ to be a constant dipath, the lemma implies that carr $|f|(z)=$ $f(\operatorname{carr} z)$ for any $z \in|X|$. 
Lemma 7. Given locally finite non-selflinked precubical sets $X, Y$, a morphism $f: X \rightarrow Y$, a dipath $q: \vec{I} \rightarrow|Y|$, and a carrier sequence $\left(x_{1}, \ldots, x_{n}\right)$ in $X$ such that carrs $q=\left(f\left(x_{1}\right), \ldots, f\left(x_{n}\right)\right)$, then there exists a dipath $p: \vec{I} \rightarrow|X|$ such that $\operatorname{carrs} p=\left(x_{1}, \ldots, x_{n}\right)$ and $q=|f| \circ p$.

Note again the implication of the lemma for constant dipaths: If $x \in X$ and $z^{\prime} \in|Y|$ are such that carr $z^{\prime}=f(x)$, then there exists $z \in|X|$ such that $\operatorname{carr} z=x$ and $z^{\prime}=|f|(z)$.

\section{Bisimulation and Dipaths}

In this final section we again fix a labeling cubical set $L$ and work in the category of higher-dimensional automata over $L$. Recall that in this category, all morphisms are non-contracting.

First we note the following stronger variant of Lemma 2 , which follows by an easy induction argument.

Lemma 8. A morphism $f: X \rightarrow Y$ is CPath-open if and only if it satisfies the property that for any reachable $x_{1} \in X$ and for any computation path $\left(y_{1}, \ldots, y_{n}\right)$ in $Y$ with $y_{1}=f\left(x_{1}\right)$, there is a computation path $\left(x_{1}, \ldots, x_{n}\right)$ in $X$ such that $y_{i}=f\left(x_{i}\right)$ for all $i=1, \ldots, n$.

We call a HDA $* \stackrel{i}{\longrightarrow} X$ special if the cubical set $X$ is freely generated by a locally finite non-selflinked precubical set, and for the rest of this section we assume our HDA to be special. Note that this is not a severe restriction: Local finiteness is hardly an issue, and the requirement on a precubical set to be non-selflinked is a natural one which is quite standard in algebraic topology, cf. [1-Def. IV.21.1].

A point $z \in|X|$ in the geometric realisation of a HDA $* \stackrel{i}{\longrightarrow} X$ is said to be reachable if there exists a dipath $p: \vec{I} \rightarrow|X|$ with $p(0)=|i *|$ and $p(1)=z$. This notion of "geometric" reachability is closely related to the one of computation path reachability defined in Section 7

Proposition 3. A point $z \in|X|$ in the geometric realisation of a special HDA $* \stackrel{i}{\longrightarrow} X$ is reachable if and only if carr $z$ is reachable.

We can now prove the main result of this article, linking bisimulation of HDA with a dipath-lifting property of their geometric realisations:

Theorem 1. Given a morphism $f: X \rightarrow Y$ of two special HDA, then $f$ is CPath-open if and only if, for any reachable $z \in|X|$ and for any dipath $q: \vec{I} \rightarrow|Y|$ such that $q(0)=|f|(z)$, there is a dipath $p: \vec{I} \rightarrow|X|$ filling in the diagram 


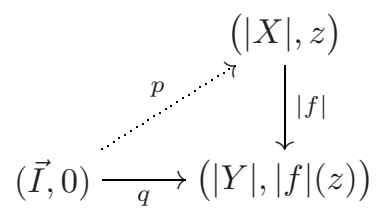

In the special case that all cubes in $X$ are reachable, we can identify $z$ with the mapping $z: 0 \mapsto z \in|X|$ and draw the above diagram in a more familiar fashion as

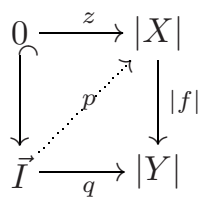

That is, a morphism $f$ from a reachable special HDA $X$ to a special HDA $Y$ is CPath-open if and only if its realisation has the right-lifting property with respect to the inclusion $0 \hookrightarrow \vec{I}$.

Proof. The morphism $f$ is non-contracting, hence it is the image of a precubical morphism, also denoted $f$, under the free functor. Assume first $f$ to be CPathopen, let $z \in|X|$ be reachable and $q: \vec{I} \rightarrow|Y|$ a dipath with $q(0)=|f|(z)$. Turn carrs $q$ into a computation path $\left(y_{1}, \ldots, y_{n}\right)$. Let $x_{1}=$ carr $z$, then $x_{1}$ is reachable, and $y_{1}=\operatorname{carr}|f|(z)=f\left(x_{1}\right)$.

We can invoke Lemma 8 to get a computation path $\left(x_{1}, \ldots, x_{n}\right)$ in $X$ such that $\left(y_{1}, \ldots, y_{n}\right)=f\left(x_{1}, \ldots, x_{n}\right)$. Lemma 7 then provides a dipath $p: \vec{I} \rightarrow|X|$ such that $q=|f| \circ p$. The construction in the proof of Lemma 7 implies that $p(0)=z$.

For the other direction, assume $|f|$ to have the dipath lifting property of the theorem, let $x_{1} \in X$ be reachable, $y_{1}=f\left(x_{1}\right) \in Y$, and let $\left(y_{1}, \ldots, y_{n}\right)$ be a computation path in $Y$.

Let $q: \vec{I} \rightarrow|Y|$ be the dipath associated with $\left(y_{1}, \ldots, y_{n}\right)$ as given by Lemma 5. Then carr $q(0)=f\left(x_{1}\right)$, thus we have $z \in|X|$ such that carr $z=x_{1}$ and $q(0)=|f|(z)$. By Proposition 3 the point $z$ is reachable, implying that we have a dipath $p: \vec{I} \rightarrow X$ such that $q=|f| \circ p$ and $p(0)=z$.

Let $\left(x_{1}, \ldots, x_{n}\right)=\operatorname{carrs} p$, then $y_{i}=f\left(x_{i}\right)$ by Lemma 6. We show that $\left(x_{1}, \ldots, x_{n}\right)$ is actually a computation path; this will finish the proof. Assume $x_{i} \triangleleft^{-} x_{i+1}$, i.e. $x_{i}=\delta_{j_{1}}^{0} \cdots \delta_{j_{\ell}}^{0} x_{i+1}$ for some sequence of indices. Then $y_{i}=$ $\delta_{j_{1}}^{0} \cdots \delta_{j_{\ell}}^{0} y_{i+1}$, but $\left(y_{1}, \ldots, y_{n}\right)$ is a computation path, hence as $Y$ is non-selflinked, the sequence of indices contains only one element $j_{\ell}$, and $x_{i}=\delta_{j_{\ell}}^{0} x_{i+1}$. Similar arguments apply to the other case.

\section{Conclusion and Future Work}

We have in this article introduced some synchronisation operations for higherdimensional automata, notably tensor product, relabeling, and restriction. 
Whether these operations capture the full flavour of HDA synchronisation remains to be seen; some other primitives might be needed. Recent work by Worytkiewicz [24]] suggests some directions.

We have also defined a notion of bisimulation for HDA which is closely related to van Glabbeek's [10] computation paths. The notion of bisimulation also defined in 10 appears to be weaker than ours, and their relation should be worked out in detail.

The notions of computation paths defined in Cattani-Sassone's [4] and in 24 differ considerably from van Glabbeek's, and as a consequence they arrive at different concepts of bisimulation and even simulation. These differences need to be worked out, and also the apparent similarities between [4] and 24.

We have shown that our notion of bisimulation has an interpretation as a dipath-lifting property of morphisms, making the problem of deciding bisimilarity susceptible to some machinery from algebraic topology. In topological language, a dipath-lifting morphism is a weak kind of fibration, hinting that fibrations (well-studied in algebraic topology) could have applications, as well. This also suggests that a general theory of directed fibrations should be developed.

We believe that our bisimulation notion should be weakened, also taking equivalence of computation paths [10] into account. We plan to elaborate on this in a future paper, and we conjecture that this bisimulation-up-to-equivalence has a topological interpretation as a property of lifting dipaths up to directed homotopy. This weaker bisimulation looks to be closely related to van Glabbeek's, and there appears to be a strong connection between his unfoldings of HDA and directed coverings of local po-spaces [7].

\section{References}

1. Glen E. Bredon. Topology and Geometry. Springer-Verlag, 1993.

2. Ronald Brown and Philip J. Higgins. On the algebra of cubes. Journal of Pure and Applied Algebra, 21:233-260, 1981.

3. Ronald Brown and Philip J. Higgins. Tensor products and homotopies for $\omega$ groupoids and crossed complexes. Journal of Pure and Applied Algebra, 47:1-33, 1987.

4. Gian Luca Cattani and Vladimiro Sassone. Higher dimensional transition systems. In Proc. LICS'96, pages 55-62. IEEE Press, 1996.

5. Sjoerd Crans. On Combinatorial Models for Higher Dimensional Homotopies. PhD thesis, Utrecht University, 1995.

6. Ulrich Fahrenberg. A category of higher-dimensional automata. Technical Report R-2005-01, Department of Mathematical Sciences, Aalborg University, 2005. http://www .math.aau.dk/research/reports/R-2005-01.ps.

7. Lisbeth Fajstrup. Dicovering spaces. Homology, Homotopy and Applications, $5(2): 1-17,2003$.

8. Lisbeth Fajstrup. Dipaths and dihomotopies in a cubical complex. Report R-2003-22, Department of Mathematical Sciences, Aalborg University, 2003. http://www.math.aau.dk/research/reports/R-2003-22.ps. Submitted to $A d$ vances in Applied Mathematics. 
9. Lisbeth Fajstrup, Eric Goubault, and Martin Raussen. Algebraic topology and concurrency. Report R-99-2008, Department of Mathematical Sciences, Aalborg University, 1999. http://www.math.aau.dk/research/reports/R-99-2008.ps. Conditionally accepted for publication in Theoretical Computer Science.

10. Robert Jan van Glabbeek. Bisimulations for higher dimensional automata. Email message, 1991. http://theory.stanford.edu/ ${ }^{\sim r v g / h d a}$

11. Robert Jan van Glabbeek. On the expressiveness of higher dimensional automata. Preprint, 2004. http://www.cse.unsw.edu.au/ ${ }^{\sim}$ rvg/hda.pdf

12. Eric Goubault. The Geometry of Concurrency. PhD thesis, Ecole Normale Supérieure, Paris, 1995. http://www.di.ens.fr/ goubault/papers/these.ps.gz

13. Eric Goubault. Labelled cubical sets and asynchronous transition systems: an adjunction. In Preliminary Proceedings CMCIM'02, 2002. http://www.di.ens.fr/ ${ }^{\sim}$ goubault/papers/cmcim02.ps.gz.

14. Marco Grandis. Directed homotopy theory I. Cahiers de Topologie et Géométrie Différentielle Catégoriques, 44:281-316, 2003. Preprint available as http://arxiv.org/abs/math.AT/0111048.

15. Marco Grandis. Directed homotopy theory II. Theory and Applications of Categories, 14:369-391, 2002.

16. Marco Grandis. Directed combinatorial homology and noncommutative tori. Math. Proc. Cambridge Philos. Soc., 2004. to appear. Preprint available as http://www.dima.unige.it/ ${ }^{\sim}$ grandis/Bsy.pdf

17. Marco Grandis and Luca Mauri. Cubical sets and their site. Theory and Applications of Categories, 11(8):185-211, 2003.

18. J. F. Jardine. Cubical homotopy theory: a beginning. Preprint, 2002.

19. André Joyal, Mogens Nielsen, and Glynn Winskel. Bisimulation from open maps. Information and Computation, 127(2):164-185, 1996.

20. William S. Massey. A Basic Course in Algebraic Topology, volume 127 of Graduate Texts in Mathematics. Springer-Verlag, 1991.

21. Robin Milner. Communication and Concurrency. Prentice Hall, 1989.

22. Jean-Pierre Serre. Homologie singulière des espaces fibrés. $\mathrm{PhD}$ thesis, Ecole Normale Supérieure, 1951.

23. Glynn Winskel and Mogens Nielsen. Models for concurrency. In Samson Abramsky, Dov M. Gabbay, and Thomas S.E. Maibaum, editors, Handbook of Logic in Computer Science, volume 4, pages 1-148. Clarendon Press, Oxford, 1995.

24. Krzysztof Worytkiewicz. Synchronization from a categorical perspective. Preprint, 2004. http://arxiv.org/abs/cs.PL/0411001. 